In spite of having pre-op 3D scans, locating the FB proved to be challenging

4 intraoperatively. Ultimately, a marker needle, in conjunction with an intraoperative $\mathrm{C}$-arm and parallax technique, allowed FB position triangulation. Blunt dissection was carried out around the needle to successfully retrieve the interdental brush (Fig. 2).

C. Frazer-Cox, D. Kumar, North Wales, UK https://doi.org/10.1038/s41415-020-2133-2

\section{Go with your gut}

Sir, the dental and maxillofacial team were asked to assess a 12-year-old male following the discovery of primary teeth in his ileostomy bag (Figs 1 and 2). The loss of teeth had not been noted prior to discovery. The patient has a medical history consisting of cerebral palsy, scoliosis and epilepsy, as well as being tracheostomy dependent.

The mechanism of ingestion is thought to have resulted from an epileptic seizure dislodging mobile primary teeth that were close to exfoliation. A dental assessment was conducted revealing a partial mixed primary-adult dentition. Chest and plain abdomen film radiographs were conducted to rule out ingestion of any further teeth.
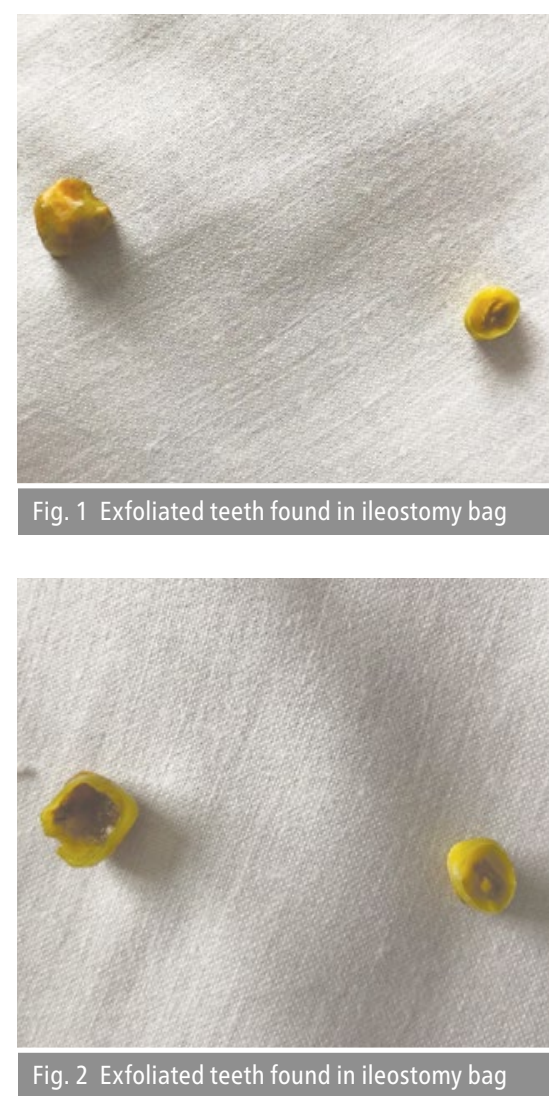

As far as the authors are aware there are no recorded cases in the literature of teeth being recovered from a stoma bag. In this case there were no adverse effects; however, ingestion of teeth could have resulted in occlusion of the stoma. This patient has a tracheostomy, and as such his airway is relatively protected, though it is not a cuffed tube. However, in patients who do not have a tracheostomy, the exfoliated teeth could have been inhaled rather than swallowed, with the potential sequelae of pneumonia or a lung abscess.

For children with reduced neuromuscular control in the mixed dentition (age 6-14 years), particularly those with vulnerable airways, consideration should be given to dental assessment and vigilance of mobile teeth, allowing for planned extraction of teeth posing an aspiration or ingestion risk.

A. Davies, J. Indoe, D. Laraway, Liverpool, UK https://doi.org/10.1038/s41415-020-2134-1

\section{Histopathology}

\section{Reconsidering syphilis}

Sir, although broadly considered a predominantly historical disease, syphilis is on the ascendancy and dentists may be wellplaced to aid in its diagnosis.

A 31-year-old male attended the oral and maxillofacial out-patient clinic for oral ulceration. Due to the COVID-19 pandemic this referral had been made following a virtual consultation only with his GP. The patient expressed a high level of concern, reporting ulcers which appeared and resolved, in different oral mucosal sites for the previous two weeks. Medical history revealed HIV, with full virological suppression. He reported a current attempt to quit smoking and feeling additional stress due to the pandemic. Recent blood tests demonstrated low serum folate, which he had commenced supplementation for in the previous days; haematinics were otherwise normal.

Oral examination revealed multiple aphthous ulcers but no suspicious mucosal abnormalities otherwise. The patient requested diagnostic biopsy stating he had 'health anxieties', despite reassurance against overt clinical malignancy. Due to COVID, his results were to be discussed by telephone but the patient was subsequently noncontactable. A letter was therefore dictated, explaining that the histopathology demonstrated non-specific inflammatory changes.

Interestingly, a colleague in sexual health saw the patient, who had subsequently self-presented to their clinic. Examination revealed multiple mouth ulcers, as noted on OMFS examination, but also a single ulcerated mucosal abnormality with a rolled margin on his left oropharynx, in keeping with primary syphilitic chancre. Syphilis serology was positive and he was treated for primary syphilis with IM benzathine penicillin. The histopathology slides were then reviewed and demonstrated a lymphoplasmacytic infiltrate.

Given the history and examination findings the OMFS differential diagnosis was recurrent aphthous stomatitis. Tissue was therefore sent for histopathological investigation and PCR was not requested. In view of the definitive diagnosis, immunohistochemistry was requested and was positive for Treponema palladium, which is a highly invasive spirochaete bacterium causing syphilis. ${ }^{1}$

This demonstrates some of the issues which arise due to patient non-disclosure of important history. The patient disclosed to the sexual health clinic that he had a number of new unprotected male partners in recent months and had his own suspicions about syphilis but did not volunteer this during his OMFS appointment. It is usual for enquiries about sexual history to be made in the sexual health clinic but this may be an area outside of the 'comfort zone' in OMFS or general dental practice.

The sexual health consultant advised syphilis has had a rise again, particularly in male homosexual groups, but that there has also been a rise in heterosexual syphilis. In many cases, clinical manifestations of syphilis are highly characteristic and given there are oral manifestations, dentists are well placed to aid in diagnosis.

A. Cameron, Bath, UK

\section{Reference}

1. Radolf J D. Treponema. In: Baron S (ed). Medical microbiology. 4th edition. Chapter 36. Galveston (TX): University of Texas Medical Branch at Galveston, 1996. Available at: https://www.ncbi.nlm.nih.gov/ books/NBK7716/ (accessed August 2020).

https://doi.org/10.1038/s41415-020-2135-0 